\title{
Co-producing knowledge in health and social care research: reflections on the challenges and ways to enable more equal relationships
}

Researchers are increasingly encouraged to co-produce research, involving members of the public, service users, policy makers and practitioners in more equal relationships throughout a research project. The sharing of power is often highlighted as a key principle when co-producing research. However, health and social care research, as with many other academic disciplines, is carried out within embedded hierarchies and structural inequalities in universities, public service institutions, and research funding systems-as well as in society more broadly. This poses significant challenges to ambitions for co-production. This article explores the difficulties that are faced when trying to put ideal coproduction principles into practice. A reflective account is provided of an interdisciplinary project that aimed to better understand how to reduce power differentials within co-produced research. The project facilitated five workshops, involving researchers from different disciplines, health, social care and community development staff and public contributors, who all had experience in coproduction within research. In the workshops, people discussed how they had attempted to enable more equal relationships and shared ideas that supported more effective and equitable co-produced research. Shared interdisciplinary learning helped the project team to iteratively develop a training course, a map of resources and reflective questions to support co-produced research. The gap between co-production principles and practice is challenging. The article examines the constraints that exist when trying to share power, informed by multidisciplinary theories of power. To bring co-production principles into practice, changes are needed within research practices, cultures and structures; in understandings of what knowledge is and how different forms of knowledge are valued. The article outlines challenges and tensions when co-producing research and describes potential ideas and resources that may help to put coproduction principles into practice. We highlight that trying to maintain all principles of co-production within the real-world of structural inequalities and uneven distribution of resources is a constant challenge, often remaining for now in the realm of aspiration. 


\section{Introduction}

o-production of research-where researchers, practitioners and members of the public collaborate to develop research together-is promoted as a way to strengthen public involvement, and create and implement more relevant and applicable knowledge, that is used in practice (Staniszewska et al., 2018; Hickey et al., 2018). Academic disciplines and funding bodies define the concept of co-production differently, using divergent methods and theories (Facer and Enright, 2016), with subsequent debate about what co-production is and who may be doing it 'properly'. We use the INVOLVE definition and principles of co-producing research (Box 1) (Hickey et al., 2018), which includes the often-agreed principle to share power more equally between partners. However, the extent to which this is achievable within structural inequalities and institutional hierarchies is debatable (Flinders et al., 2016).

This commentary article reflects on a project that aimed to:

- share interdisciplinary learning about co-produced research

- understand how to enable more equal relationships with coproduction partners, particularly public contributors-defined as members of the public including patients, potential patients, carers and people who use health and social care services (in contrast to people who have a professional role in health and social care services or research) (NIHR CED, 2020).

- develop training and resources to support co-produced research.

The project was developed by a team of three applied health researchers, a public involvement lead and three public contributors (with in-depth experiences of co-produced research) undertaken within the National Institute for Health Research (NIHR) Applied Research Collaboration (ARC) West, an organisation that develops applied health and social care research. Through facilitating five project workshops, we engaged with eleven researchers from five disciplines; six practitioners; and eleven public contributors with involvement and co-produced research experiences. We shared practical lessons across disciplinary boundaries about how to do coproduced research more equitably (Oliver and Boaz, 2019). These workshops helped the project team progressively and iteratively develop a training course, a map of resources (Farr et al., 2020) and reflective questions (Davies et al., 2020), freely available to support co-produced research.

This article explores the extent to which these multidisciplinary lessons can help us transform knowledge production in more equitable ways, outlining our learning from this project. First, we overview some conceptual issues with the use of the word 'co-production'. We then discuss key matters raised in our interdisciplinary workshops: 'Who is involved and when in co-produced research?'; 'Power dynamics within health and social care research'; and 'Communication and relationships'. We conclude by highlighting that bringing co-production principles into the real research world is fraught with difficult and messy compromises. Researchers (often lower in the academic hierarchy) may be caught up in battling systems and policies to enable co-production to happen, especially where they attempt to address issues of power and control within the research process.

\section{The concept of co-production}

The NVOLVE co-production principles (Box 1) (Hickey et al., 2018) build on public policy co-production literature (Boyle and Harris, 2009; Staniszewska et al., 2018), which explores how service users can take an active role within the provision of public services (Brudney and England, 1983; Ostrom, 1996). A key premise is that service users have a fundamental role in producing services and outcomes that are important to them (Brandsen and Honingh, 2016). While in our project we particularly wanted to focus on ways of sharing power with service users and public contributors, defining who is involved in co-produced research varies across disciplines. The active involvement of service users/members of the public has sometimes been lost in research that is labelled as co-produced. UK funding councils such as the Economic and Social Research Council (ESRC) talk of co-produced research as developed between academic and non-academic organisations or communities (Campbell and Vanderhoven, 2016; ESRC, 2019). In health services research, authors have used the non-specific language of 'stakeholders' (Oliver et al., 2019). Sometimes the main research co-production partner has been practitioners (Heaton et al., 2016) and service users have been relegated to 'context' (Marshall et al., 2018), rather than being active agents and partners. This obfuscation of the role of service users/ members of the public in co-production is detrimental to the drive for inclusivity, democratisation and equity within co-produced research, which addresses the needs of service users/ marginalised citizens (Williams et al., 2020), overlooking the long political history of demands from service users to be more democratically involved in health and social care services and research (Beresford, 2019).

In our project, we particularly wanted to focus on how to equalise relationships with service users and public contributors (defined above) within co-produced research. The egalitarian and democratic principles of co-production means that service users, who may have been marginalised and are on the receiving end of professional 'expertise', now become equal partners in research (Williams et al., 2020). Best practice in co-produced research remains contested, with a significant theory-practice gap (Lambert and Carr, 2018). We wanted to understand what practices and resources could help bring principles into practice, when we are working within a context of structural inequalities.

\section{Who is involved and when in co-produced research?}

INVOLVE states that co-production should 'occur from the start to the end of the project' (Hickey et al., 2018) (p. 4). The principle

\section{Box 1: | INVOLVEa Definitions and principles of co-produced research (Hickey et al., 2018)}

'Co-producing a research project is an approach in which researchers, practitioners and the public work together, sharing power and responsibility from the start to the end of the project, including the generation of knowledge' (p. 4).

Principles include:

- Sharing power where research is owned by everyone and people are working together in more equal relationships

- Including all perspectives and skills to ensure that everyone who wants to make a contribution can do so, with diversity, inclusiveness and accessibility being key

- Respecting and valuing the knowledge of everyone, with everyone being of equal importance, and benefitting from the collaboration

- Reciprocity and mutuality, building and maintaining relationships and sharing learning

- Understanding each other with clarity over people's roles and responsibilities.

aINVOLVE supported active public involvement in NHS, public health and social care research, with a new NIHR Centre for Engagement and Dissemination (CED) launched in April 2020. 
Table 1 Navigating challenges relating to who is involved, and when.

\section{Challenges}

Involving the public in the generation of research ideas

Developing funding applications with public contributors and community groups

Decision-making within the group

Using co-production principles throughout the project, from beginning to end

Consider training needs at the start and throughout the project

\section{Helpful practices and resources}

- Some Universities may provide early public engagement seed funds that can be used to pay for public contributors' time to help develop funding applications.

- The James Lind Alliance conduct research priority setting partnerships to identify and prioritise questions informed by the public's and clinicians' views. With limited resources, these are useful to inform research priorities.

- Encourage early discussions with public contributors to increase their influence. Be creative in the ways you get ideas from people.

- Talk to members of the public, patient representatives and community groups before developing applications, to include wider perspectives and ensure community group and public contributor time is appropriately costed in.

- Discuss roles and responsibilities before funding applications are submitted, so that the budget and resources are sufficiently allocated to non-academics within the project. Ensure this includes time to work actively on key project tasks.

- Clarify and agree how decisions will be made in the group. Explore the potential for all to play an active role in directing the research and shaping the projects. Play to people's strengths and preferences. Discuss and clarify leadership role/style for the group and who has responsibility for which aspects of it. Do people have sufficient resource and power to take on these responsibilities? Do they need support or training?

- Be clear with those involved about areas of focus and constraints if these are not negotiable.

- Public contributors may need explanations/information about University systems and how long things might take, including requirements for ethics permission etc. They may also need topic or context-specific background information to support inclusion.

- Use a pragmatic approach, where only some parts of the research project are co-produced, if facing external constraints (INVOLVE, 2019). Identify which aspects of the research cycle can be co-produced and how can this be supported.

- Include early discussions about what the project might produce including academic papers, clear short summaries, who might be authors, and how to share what is co-produced.

- Public contributors should be given appropriate training to enable them to understand the research and contribute to discussions. They may not wish to get involved in all aspects of the research process in detail so training should be tailored to individual needs and preferences, and reviewed regularly as the project progresses.

- Both formal training courses and informal training on-the-job training may be important. Informal mentoring and feedback can be helpful.

- Researchers may also have additional training needs as co-produced research needs a wide range of skills. to include all perspectives and skills-make sure the research team includes all those who can make a contribution' (Hickey et al., 2018) (p. 4) can be highly aspirational.

In our interdisciplinary workshops we discussed how there is often a lack of funding to pay public contributors to help develop a funding application. Formative ideas about research priorities and design can often be made by researchers before other people are involved. Our workshop discussions noted that involving all stakeholders who potentially have an interest in a project could be a very large and diverse group. It would be difficult to involve everyone, and this could be in tension with the idea that smaller groups can work better together. There are usually practical constraints on team numbers, budgets to pay for public contributors' time and project scale and size. A tension can exist between the number of people you can viably include, and the diversity of the group you are working with. More generally, workshop participants highlighted problematic issues of claims to representation, where people within a co-production group need to be aware that they don't speak for everyone-not even everyone in a group they are there to 'represent'-and there was a need to look for opportunities to draw other perspectives in.

Workshop discussions included that when public contributors join a project there is a need to support people to take on different roles, and for people to also have choice and work from their strengths, rather than assuming that everyone has to do everything. Some group members may feel they lack skills or expertise in particular areas, and so may need training, support and mentoring. There may also be assumptions about who is going to do what work, which may need to be explicitly discussed and agreed. Ensuring proper payment of public contributors is an essential element of co-production. If public contributors are going to collect research data, they need appropriate payment, contracts and to follow all research governance processes. Within UK National Health Service (NHS) research that may mean having Research Passports, Good Clinical Practice training and Disclosure and Barring Service checks, if they are working with vulnerable people or children. Not all these processes are designed for public contributors, they can be potentially problematic to navigate, and researchers may need to support public contributors through this process. Table 1 summarises some of the challenges around who is involved, and when in co-produced research, and potential practices and resources that may help.

\section{Power dynamics within health and social care research}

Theoretical perspectives on power. Critical and interdisciplinary perspectives on power can help us understand how to facilitate more equitable partnerships within research and co-produced work (Farr, 2018; Oliver and Boaz, 2019). The first principle of coproduction is to share power through 'an equal and reciprocal relationship' between professionals and people using services (Boyle and Harris, 2009) (p. 11). However, several authors highlight how co-production can be a rhetorical device to hide power and social inequities (Flinders et al., 2016; Thomas-Hughes, 2018). Using Lukes (2005) dimensions of power, Gaventa (2007) conceives that power can be visible (institutions, structures, resources, 
rules), hidden (agenda-setting, some voices more dominant within decision-making), or invisible (embedded in beliefs and language).

Focussing first on visible aspects, structural and resource issues can impinge on people's ability to co-produce, for example funders' top-down control of research priorities and funding streams, alongside NHS and government political priorities. University research environments can be competitive, 'unkind and aggressive', which can crowd out 'collegiality and collaboration' (WellcomeTrust, 2020), exactly the kind of principles that academics are being encouraged to adopt through co-production. Traditional research frameworks are ill-fitted to the challenges of transforming power and control that are needed for co-productive practice (Lambert and Carr, 2018). Power hierarchies are intrinsic to research processes, with people experiencing competing expectations (from public contributors and communities, co-researchers, colleagues and institutions) when working in this way (Lenette et al., 2019). How do researchers create co-production circles of equality, reciprocity and share power with public contributors, when often researchers themselves are on temporary contracts and subject to the pressures of publishing, funding, impact and self-promotion within 'toxic' (Wellcome Trust, 2020) competitive structures? Understanding who is involved and how in decision-making processes (hidden aspects of power) is essential to understand how power is exercised. However, political scientists have long ago illustrated that 'even the most internally democratic small collectives cannot in fact achieve equality of power in their decisions' (Mansbridge, 1996, p. 54).

Scrutinising invisible aspects of power, power can be seen to operate through knowledge, social relations and the language we use (Foucault, 1977). The principle of respecting and valuing the knowledge of all (Hickey et al., 2018) can be challenging in a healthcare context where a knowledge hierarchy with traditional positivist epistemological assumptions values an 'unbiased, objective' position. Co-productive approaches can be grounded in critical theory (Bell and Pahl, 2018; Facer and Enright, 2016), as opposed to traditional scientific paradigms. The experiential contextualised and tacit knowledge of people who use services, and related qualitative and participatory action methods, can be valued less than knowledge derived from randomised controlled trials (RCTs). This increases the challenge of co-production, as the values and methods of health and social care research may align less with co-production principles. Indeed the very idea that coproduction and the sharing of power can actually happen within mainstream University spaces has been contested, with Rose and Kalathil (2019) arguing that Eurocentric hierarchical institutions that privilege rationality and reason will never be coming from a place where different knowledges are valued equally.

Understanding power in practice. In our project it was difficult to maintain a focus on power relations in the face of a strong tendency to emphasise practicalities, highlighting the difficulties of bringing these issues into clearer focus. An analysis of power dynamics may be an important aspect of a sociological study, but not one considered of such importance within health and social care research.

Focussing first on visible, structural aspects of power, workshop participants discussed their experience that within research that is formally 'owned' by a University (i.e., the Principal Investigator (PI) legally responsible for the project is situated within a University) there are associated issues of accountability and formal responsibility for delivering a funded research project. This creates constraints where projects have to deliver what is described rather than what emerges from the co-production process. How a PI works to develop a collaborative leadership style is an under-researched area. Within our own project we all held some unspoken assumptions about leadership and ensuring progress toward our project objectives. Workshop participants highlighted that organisational systems may not support co-production (e.g., finance, human resources and funding systems) so researchers may have to be tenacious to advocate for system changes in order to achieve things, which can be frustrating and timeconsuming. For these myriad reasons, realistic resourcing of researcher time for co-production is needed, and many researchers may still end up putting discretionary time into projects to make coproduction a success. There are few tools to help researchers avoid or alleviate risks to themselves and their stakeholders, such risks including practical costs, personal and professional costs to researchers, and costs to stakeholders (Oliver et al., 2019).

In relation to decision-making, workshop participants noted that in a pragmatic sense, doing everything by committee and consensus can impede project progress, as no decisions can be made until everyone is present at meetings. Even if decisions are made with everyone present, the power dynamics between people does not necessarily ensure that decisions are shared and agreed by everyone. Within our own project, where we were trying to stick to the principles of co-production, we found that we often had discussions between paid staff members outside of team meetings where thinking was developed and decisions taken. If public contributors are without employment contracts and are not working alongside other staff, there is potential for them to be excluded from informal discussions and decisions in day-to-day tasks. In our workshops there were discussions about whether researchers needed to 'get out of the way' and 'sit on their hands' in order to make space for others. We discussed how to practically create space for diverse knowledge and skills to be shared and considered whether it is possible to identify shared interests or if there is always a political struggle for power.

Through our project, we reflected as a team how assumptions and practices of how we do healthcare research may be deeply embedded within academic cultures. This links with Foucault's perspectives on power dynamics (Foucault, 1977), every act and assumption we make is imbued with power, which makes power particularly hard to observe, grasp, critique, challenge and transform. We all have subconscious beliefs and work within cultural assumptions, thus continual critical reflective practice, and constant attention to fluctuating power relations is needed (Farr, 2018; Bell and Pahl, 2018). In workshops, suggested ways to address cultural issues included harnessing the current trends for co-production and using this to start challenging engrained cultures and accepted ways of doing things. Current funder prioritisation of co-production can enable senior researcher support for co-production, as organisational leaders recognise the cultural capital of the word and practices of ' $\mathrm{co}-$ production'. Raising awareness of NIHR and other policy commitments to co-production may be a useful influencing strategy to engage more senior staff, as organisational support can be crucial to facilitate co-production. However, there is always the risk of tokenism and rhetoric (Flinders et al., 2016; Thomas-Hughes, 2018).

We considered within our project that the relationships between personal experiential knowledge, practice-based knowledge of healthcare staff, and dominant healthcare research need to be better understood if we are to co-produce knowledge together. We reflected on whether the aim of co-production projects is to modify the knowledge hierarchy completely, or to bring in experiential expertise/lived experience to influence the knowledge production process so the knowledge produced is more practical/ effective/implementable. This second, more limited aim of making evidence more co-productively, so that it is more useful in practice may be more achievable, whereas modifying the dominant knowledge hierarchy was beyond our scope and influence.

\section{Communication and relationships}

The above dimensions of power (Lukes, 2005) have been augmented and brought together into a broader theoretical framework 
(Haugaard, 2012), which also incorporates 'power with' (Arendt, 1970), where emancipatory power can be harnessed through our 'capacity to act in concert'. Arendt's work highlights how we can collectively use our power together in more empowering ways. This links with a key principle of co-production, reciprocity, where everyone benefits from working together.

Consideration of what different team members want from working together, and therefore what reciprocity means within a project is needed. We discussed in the interdisciplinary workshops how the kind of benefits wanted by public contributors might include developing skills, confidence and work experience, and meeting such expectations may not usually be considered as research aims. Through our project we saw how co-production is strongly reliant on good communication and relationships. Strong facilitation and chairing skills are needed within meetings, to encourage everyone to contribute and challenge unhelpful behaviours, e.g., using jargon, or one person speaking a lot to the exclusion of others. People in our workshops discussed how some public contributors might need additional support to get more involved, e.g., having premeetings to help people get to grips with some information and/or issues, or the provision of materials in different accessible formats. If a co-production project includes people with specific communication needs, the group may need additional time and skills to be able to offer ways of working that are suitable for all. The NIHR is encouraging researchers to involve communities and groups that are often excluded. This means more outreach work to go out and meet with people in the places that they find accessible and comfortable, which can include project meetings in community locations, which may require additional resources.

Developing relationships and trust between team members may take time and requires emotional work. In our workshops we discussed how if the public contributor role includes sharing personal experiences for the benefit of the project, then researchers may also need to drop the 'professional' mask and share more personally and expose their own vulnerabilities (Batalden, 2018) to support more equal relationships. The challenges of university structural hierarchies were also discussed, including how it was often the responsibility of more 'junior' (i.e., lower in the hierarchy) researchers, and often women, to do the relational work (Lenette et al., 2019). Senior researchers do not necessarily understand the implications of co-production, for example one person shared how their Principal Investigator assumed that having a public contributor on the team would increase capacity and speed work up, unappreciative of the extra time needed for support, training and communication, including at the weekends, when public contributors could be carrying out work. Meeting the support and learning needs of team members can be challenging, both for researchers and public contributors, as co-produced research may take researchers outside the skills and knowledge usually expected in their professional environment. Even when these needs within a coproduction project are recognised, research funders may not understand the resource and capacity implications.

A key element of running a co-production project identified within our work was the ongoing need for time to reflect on group processes to support and maintain different ways of working. Finding time for reflection can be challenging alongside creating an environment where everyone can honestly reflect on what it's like to be in the group. This requires strong facilitation skills, particularly if there are tensions and conflicts. Addressing communication and relationship challenges are key to developing and sustaining a sense of shared ownership, and we outline some helpful practices in Table 2.

\section{Reflections on our own attempts at co-production}

The conception of our project came initially from conversations between a researcher and public involvement specialist with previous experience as a service user and user-controlled research, wanting to create a space to share interdisciplinary learning between everyone about co-production. It could be argued that as the generation of the idea did not include public members in this first discussion it was not truly co-produced. We acknowledged that there were gaps between the lessons our project produced, and how the project itself had been carried out. It was very challenging to implement all INVOLVE principles (Hickey et al. 2018), and we question the extent to which they can ever be fully realised within our current contexts. Practically, we found that we should have allocated more resources to payment of our public contributors to take on additional roles. A focus on relationships and reflection was hard to maintain in the face of a small group trying to deliver an ambitious project to time, alongside other competing commitments. However, in our own reflective discussions we acknowledged that a sense of ongoing commitment to the project from everyone felt key to our group process and successfully getting the project done. In writing this article we met together several times to plan and develop sections, tables and points we wanted to get across. However, the actual writing tended to fall to the academics and public involvement specialist, who had more of the technical knowledge of what was expected. Demands of time, the juggling of commitments, and lack of resources meant that writing the article was not truly 'co-produced'. Indeed, through the process, a public contributor co-author said they found the reviewers' comments 'a bit overwhelming, with uncertainty of how to approach this. Another public contributor co-author expressed similar experiences with reviewers comments on another paper they had previously coauthored. The publication process can be a challenge to researchers as well, who are more familiar with these traditional academic practices.

\section{Conclusion}

Sharing power in the face of embedded hierarchies and inequalities is an obvious challenge for co-production. The gap between coproduction principles and practice is a tricky territory. Working with everyone who is interested in an issue, having a focus on meeting the priorities of communities and people we work with, and co-producing all aspects of a project from beginning to end will be difficult to deliver in many projects in health and social care research. Working directly with members of the public is likely to require more adaptation of research project processes and to 'usual ways of doing things', alongside additional time and resources. People have different skills and uneven access to resources, and people may need considerable training and support to work together more equally. However, our experience is that funders do not necessarily understand this and doing co-production on a small budget can be particularly challenging. Time investment and the emotional work required to build relationships necessary for successful co-production is both underappreciated and under-resourced. This reflects disparities in power between those who do this work and those who hold most power in universities. Recognising, recording, documenting and consistently budgeting for this work may help to make it more visible. Timing of funding is also crucial as many research teams do not have access to institutional 'core' funding, or seed funding grants, for public contributor involvement at the research development stage. As it is unlikely that most co-production projects will be able to include people with all the relevant perspectives and skills it is important to actively discuss and agree who can be involved and to be open about and discuss restrictions, which can be an act of power in itself.

Oliver and Boaz (2019) want to 'open the door' to more critical multidisciplinary accounts of evidence production and use, highlighting that some people want to direct energies to democratise knowledge for all. Interdisciplinary lessons from this project question the extent to which co-production processes can enable this, given the challenges we have highlighted. We consider that the jury is still out on the viability of co-production in the context of health and 


\section{Table 2 Navigating challenges relating to communication and relationships.}

\section{Challenges}

Reciprocity-everyone benefits

Building strong relationships

Clarifying communications

Personal disclosure and safety

Meeting learning needs

Relationships, trust and regular reflection

\section{Helpful practices and resources}

- At the start people share what they bring to the project and what they would like to get from the process.

- Clarify what can and can't be offered within a project's remit.

- At the start make time to get to know each other, including social time together, e.g., over lunch, which may change the usual ways of working.

- All meetings to include welcoming everyone and providing hospitality, refreshments etc.

- Agree ways of working in the group, including clarifying roles, values and assumptions.

- Think about facilitation/chairing of meetings and other group processes, potentially including sharing leadership roles across the team.

- Have time in all meetings for people to check in and say how they are, not just a task focused agenda.

- It might be helpful to have someone in the team who becomes a 'co-production advocate' who can remind the group about relational needs and helpful group processes.

- Agree key communication processes, e.g., how notes of meetings are being taken, when/how they are shared.

- What communication processes suit the group? Written notes might not be accessible for all. Videos or verbal recordings of notes of meetings may make key points accessible. Not everyone uses email.

- Agree feedback processes for work being done between meetings so that everyone understands enough about project progress and what is happening.

- Effective collaboration may need some group members to share a range of experiences, so relationships may differ from professional 'norms', which do not include personal disclosure.

- Disclosure of personal experiences is likely to have implications for power relations-particular if disclosure includes information about vulnerability or issues associated with stigma. Safety and trust are likely to be key in such situations, so a group may need to understand how to support and manage disclosure-including understanding how to respond to any distress or trauma that might be triggered.

- Active consideration of learning and support needs for different group members and how these might be met. This could be by providing opportunities to practice new skills, e.g., chairing, or by finding other ways to meet identified needs.

- Develop processes that work for the group to support reflection and review of how things are going.

- Hierarchical relationships may make reflective processes challenging, so consideration of how the group can support the giving of more difficult feedback may be needed.

- Make time in meetings, or have additional time together, to reflect on how the group is working and whether initial ideas about communication and relationships are being maintained and/or need development. social care research. While some (Rose and Kalathil, 2019) find the promise of co-production untenable in mental health, we hope we can find a meaningful way forward. However, 'putting what we already know [about co-production] into practice' (Oliver and Boaz, 2019) can be very challenging. Our own experiences led us to reflect that to be working toward co-production principles means that you have to consistently be challenging 'business as usual'-we consider a key point here is how to maintain sufficient self and team support to keep trying to do this in practice. Establishing reflective processes that encourage consideration of power issues are likely to be essential. Our approach to help ourselves and others navigate the challenges of coproduction has been to identify ways in which groups can start to address power issues as highlighted in Tables 1 and 2, and to develop practical freely available outputs including a map of resources and reflective questions (Farr et al., 2020; Davies et al., 2020). We need to understand more about how effective these strategies are, and whether co-production really does make a difference to the use of research. We need to encourage honest reporting of projects, their outcomes and the balance between the benefits and challenges of trying to implement the principles. However, power structures may mitigate against reporting of challenges and problems in research. Other research gaps include understanding what projects will benefit most from a co-production approach. Can co-production deliver more practical and implementable research findings, and if so how? How do we best challenge and change some of the structural inequalities within academia that impede co-production (Williams et al., 2020)? How do we integrate experiential, practice and researchbased knowledge to improve health and social care?

Our experiences on this project highlight the ongoing challenges to truly put the principles of co-production into practice. During this project we used the phrase 'I am always doing what I can't do yet in order to learn how to do it' (van Gogh, 1885), to illustrate our limitations, yet continual striving toward an ideal. The quote continues '...I'll end by saying that the work is difficult, and that, instead of quarrelling, the fellows who paint peasants and the common people would do wisely to join hands as much as possible. Union is strength...' (van Gogh, 1885). Forgiving the dated language and connotations of this quote, the principles of joining hands and facilitating union are important co-production ideals that we continually need to remember, relearn and put our hearts into practising.

Received: 28 February 2020; Accepted: 31 March 2021; Published online: 06 May 2021

\section{References}

Arendt H (1970) On violence. Allen Lane, Penguin, London

Batalden P (2018) Getting more health from healthcare: quality improvement must acknowledge patient coproduction-an essay by Paul Batalden. BMJ 362: k3617. https://doi.org/10.1136/bmj.k3617

Bell DM, Pahl K (2018) Co-production: towards a utopian approach. Int J Soc Res Methodol 21(1):105-117. https://doi.org/10.1080/13645579.2017.1348581

Beresford P (2019) Public participation in health and social care: exploring the coproduction of knowledge. Front Sociol 3(41). https://doi.org/10.3389/ fsoc.2018.00041

Boyle D, Harris M (2009) The challenge of co-production. New Economics Foundation, London

Brandsen T, Honingh M (2016) Distinguishing different types of coproduction: a conceptual analysis based on the classical definitions. Publ Admin Rev 76 (3):427-435. https://doi.org/10.1111/puar.12465

Brudney JL, England RE (1983) Toward a definition of the coproduction concept. Publ Admin Rev 43(1):59-65. https://doi.org/10.2307/975300

Campbell HJ, Vanderhoven D (2016) N8/ESRC research programme. Knowledge that matters: realising the potential of co-production. N8 Research Partnership, Manchester, https://www.n8research.org.uk/media/Final-Report-CoProduction-2016-01-20.pdf. Accessed 23 Mar 2021

Davies R, Andrews H, Farr M, Davies P, Brangan E, Bagnall D (2020) Reflective questions to support co-produced research. National Institute for Health 
Research (NIHR) ARC West and People in Health West of England, University of Bristol and University of West of England. http://bit.ly/ CoProResources. Accessed 23 Mar 2021

ESRC (2019) Guidance for collaboration. Economic and Social Research Council. https://esrc.ukri.org/collaboration/guidance-for-collaboration/. Accessed 29 Nov 2020

Facer K, Enright B (2016) Creating Living knowledge: the connected communities programme, community-university relationships and the participatory turn in the production of knowledge. University of Bristol/ AHRC Connected Communities, Bristol

Farr M (2018) 'Power dynamics and collaborative mechanisms in co-production and co-design processes'. Crit Social Policy 38(4):623-644. https://doi.org/ 10.1177/0261018317747444

Farr M, Davies R, Davies P, Bagnall D, Brangan E, Andrews H (2020) A map of resources for co-producing research in health and social care. University of Bristol and University of West of England, National Institute for Health Research (NIHR) ARC West and People in Health West of England http:// bit.ly/CoProResources. Accessed 23 Mar 2021

Flinders M, Wood M, Cunningham M (2016) The politics of co-production: risks, limits and pollution. Evid Policy 12(2):261-279. https://doi.org/10.1332/ 174426415X14412037949967

Foucault M (1977) Discipline and punish: the birth of the prison. Allen Lane, London

Gaventa J (2007) Levels, spaces and forms of power: analysing opportunities for change. In: Berenskoetter F, Williams MJ (eds) Power in world politics. Routledge, Abingdon, pp. 204-224

Haugaard M (2012) Rethinking the four dimensions of power: domination and empowerment. J Polit Power 5(1):33-54. https://doi.org/10.1080/ 2158379X.2012.660810

Heaton J, Day J, Britten N (2016) Collaborative research and the co-production of knowledge for practice: an illustrative case study. Implement Sci 11(1):20. https://doi.org/10.1186/s13012-016-0383-9

Hickey G, Brearley S, Coldham T, Denegri S, Green G, Staniszewska S, Tembo D, Torok K, Turner K (2018) Guidance on co-producing a research project. INVOLVE, Southampton

INVOLVE (2019) Co-production in action: number one. INVOLVE, Southampton

Lambert N, Carr S (2018) 'Outside the Original Remit': Co-production in UK mental health research, lessons from the field. Int J Ment Health Nurs. 27 (4):1273-1281

Lenette C, Stavropoulou N, Nunn C, Kong ST, Cook T, Coddington K, Banks S (2019) Brushed under the carpet: examining the complexities of participatory research. Res All 3(2):161-179. https://doi.org/10.18546/RFA.03.2.04

Lukes S (2005) Power: a radical view. Macmillan, London

Mansbridge J (1996) Using power/ fighting power: the polity. In: Benhabib S (ed.) Democracy and Difference. Princeton University Press, Princeton, pp. 46-66

Marshall M, Mear L, Ward V, O'Brien B, Davies H, Waring J, Fulop N (2018) Optimising the impact of health services research on the organisation and delivery of health services: a study of embedded models of knowledge coproduction in the NHS (Embedded). NIHR. https://www.journalslibrary.nihr. ac.uk/programmes/hsdr/165221/\#/. Accessed 23 Mar 2021

NIHR CED (2020) Centre for engagement and dissemination recognition payments for public contributors. https://www.nihr.ac.uk/documents/centre-forengagement-and-dissemination-recognition-payments-for-publiccontributors/24979\#1. Accessed 23 Mar 2021

Oliver K, Boaz A (2019) Transforming evidence for policy and practice: creating space for new conversations. Pal Commun 5(1):60. https://doi.org/10.1057/ s41599-019-0266-1

Oliver K, Kothari A, Mays N (2019) The dark side of coproduction: do the costs outweigh the benefits for health research? J Health Res Policy Syst 17(1):33. https://doi.org/10.1186/s12961-019-0432-3
Ostrom E (1996) Crossing the great divide: coproduction, synergy, and development. World Dev 24(6):1073-1087. https://doi.org/10.1016/0305-750X(96)00023-X

Rose D, Kalathil J (2019) Power, privilege and knowledge: the untenable promise of co-production in mental "health". Front Sociol 4:57. https://doi.org/10.3389/ fsoc.2019.00057

Staniszewska S, Denegri S, Matthews R, Minogue V (2018) Reviewing progress in public involvement in NIHR research: developing and implementing a new vision for the future. BMJ Open 8(7):e017124. https://doi.org/10.1136/ bmjopen-2017-017124

Thomas-Hughes H (2018) Ethical 'mess' in co-produced research: reflections from a U.K.-based case study. Int J Soc Res Methodol 21(2):231-242. https://doi. org/10.1080/13645579.2017.1364065

van Gogh V (1885) RE: Letter from Vincent van Gogh to Anthon Van Rappard WellcomeTrust (2020) What researchers think about the culture they work in Wellcome Trust, London, https://wellcome.org/sites/default/files/whatresearchers-think-about-the-culture-they-work-in.pdf. Accessed 23 Mar 2021

Williams O, Sarre S, Papoulias SC, Knowles S, Robert G, Beresford P, Rose D, Carr S, Kaur M, Palmer VJ (2020) Lost in the shadows: reflections on the dark side of co-production. Health Research Policy and Systems 18 (1)

\section{Acknowledgements}

This project was funded by the University of Bristol Public Engagement Seed Funding and Research Staff Development fund. It was supported by the National Institute for Health Research (NIHR) Applied Research Collaboration West (NIHR ARC West). The views expressed in this article are those of the authors and not necessarily those of the NIHR or the Department of Health and Social Care. Many thanks to everyone who attended our workshops, got involved in and supported the project to help us develop our resources and training. We couldn't have done this with you!

\section{Competing interests}

The authors declare no competing interests.

\section{Additional information}

Correspondence and requests for materials should be addressed to M.F.

Reprints and permission information is available at http://www.nature.com/reprints

Publisher's note Springer Nature remains neutral with regard to jurisdictional claims in published maps and institutional affiliations.

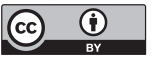

Open Access This article is licensed under a Creative Commons Attribution 4.0 International License, which permits use, sharing, adaptation, distribution and reproduction in any medium or format, as long as you give appropriate credit to the original author(s) and the source, provide a link to the Creative Commons license, and indicate if changes were made. The images or other third party material in this article are included in the article's Creative Commons license, unless indicated otherwise in a credit line to the material. If material is not included in the article's Creative Commons license and your intended use is not permitted by statutory regulation or exceeds the permitted use, you will need to obtain permission directly from the copyright holder. To view a copy of this license, visit http://creativecommons.org/ licenses/by/4.0/.

(C) The Author(s) 2021

\section{Michelle Farr (10) ${ }^{1,2}$, Philippa Davies (10 ${ }^{1,2}$, Heidi Andrews ${ }^{1,2}$, Darren Bagnall ${ }^{1,2}$, Emer Brangan (1) ${ }^{1,2,3}$ \& Rosemary Davies (iD) ${ }^{1,4}$}

${ }^{1}$ The National Institute for Health Research Applied Research Collaboration West (NIHR ARC West) at University Hospitals Bristol and Weston NHS Foundation Trust, Bristol, UK. ${ }^{2}$ Population Health Sciences, Bristol Medical School, University of Bristol, Bristol, UK. ${ }^{3}$ School of Health and Social Wellbeing, University of the West of England, Bristol, UK. ${ }^{4}$ Faculty of Health and Applied Sciences, University of the West of England, Bristol,

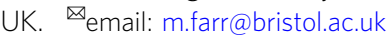

